\title{
Análise das dificuldades dos alunos de graduação diante de procedimentos clínicos de Periodontia
}

Análisis de la dificultad de los estudiantes de graduación frente a los procedimientos clínicos de periodoncia Analysis of the difficulties of undergraduate students during clinical periodontics procedures

\section{Kamila Fabro de LIMA ${ }^{1}$} Douglas FERLA ${ }^{1}$

Juliane Pereira BUTZE ${ }^{2}$

${ }^{1}$ Graduado(a) do Curso de Odontologia do Centro Universitário da Serra Gaúcha - FS, 95020-472 Caxias do Sul- RS, Brasil ${ }^{2}$ Doutora em Periodontia, Professora do Curso de Odontologia do Centro Universitário da Serra Gaúcha - FSG, 95020-472 Caxias do Sul - RS, Brasil

\section{Resumo}

Objetivos: O presente estudo avaliou as principais dificuldades relatadas pelos estudantes do último ano do Curso de Odontologia do Centro Universitário da Serra Gaúcha (FSG) na execução de procedimentos básicos de Periodontia. Materiais e Métodos: Foi aplicado aos participantes um questionário estruturado composto por 14 questões objetivas que abordavam passos relevantes do tratamento periodontal básico. O grau de dificuldade foi avaliado através da escala Likert em $0=$ nenhuma; 1 = leve; 2 = moderada; 3 = alta e 4 = muito alta. Para a análise dos dados, o grau de dificuldade foi dicotomizado em nenhuma a leve, moderada, alta e muito alta. Resultados: Participaram do estudo 46 alunos matriculados no último semestre do Curso de Odontologia do Centro Universitário da Serra Gaúcha (FSG). Marcar a perda de inserção (PI) foi o procedimento descrito como de dificuldade alta/muito alta pelos estudantes, seguidos de procedimentos de dificuldade moderada, tais como marcar a profundidade de sondagem (PS) e raspagem e alisamento radicular. Porém, anestesiar e diagnosticar gengivite foram os únicos procedimentos relatados por todos os acadêmicos (100\%) como tendo nenhuma dificuldade. Conclusão: De acordo com os resultados obtidos no presente estudo, pode-se concluir que os procedimentos periodontais relatados como os de maiores dificuldades pelos estudantes do Curso de Odontologia do Centro Universitário da Serra Gaúcha (FSG) são os procedimentos de diagnóstico de periodontite e perda de inserção periodontal.

Descritores: Odontologia; Ensino; Periodontia.

\section{Abstract}

Objectives: This study assessed the main difficulties reported by final year students of the University of Serra Gaúcha (FSG), Dentistry Faculty during the implementation of basic periodontal procedures. Materials and Methods: We applied to the participants a structured questionnaire with 14 objective questions that addressed relevant steps of basic periodontal treatment. The degree of difficulty was evaluated by Likert scale $0=$ none, $1=$ mild, $2=$ moderate, $3=$ high and $4=$ very high. For the data analysis, the degree of difficulty was dichotomized in any mild, moderate, and high and very high. Results: The study included 46 students enrolled in the last semester of Dentistry Faculty of the University of Serra Gaúcha (FSG). Marking the clinical attachment loss (CAL) was the procedure described as High / Very High difficulty by students, followed by moderate difficulty procedures. However, anesthetize and diagnosing gingivitis were the only procedures reported by all students (100\%) as performed having no difficulty. Conclusion: According to the results obtained in this study, it can be concluded that periodontal procedures reported as the greatest difficulties by students of Dentistry faculty of the University of Serra Gaúcha (FSG), are the periodontitis diagnostic procedures and displaying the clinical attachment loss.

Descriptors: Dentistry; Teaching; Periodontics.

\section{Resumen}

Objetivos: Este estudio evaluó las principales dificultades reportadas por los estudiantes del último año del Curso de Odontología del Centro Universitário da Serra Gaúcha (FSG) en la ejecución de procedimientos básicos de Periodoncia. Materiales y métodos: se aplicó a los participantes un cuestionario estructurado compuesto por 14 preguntas objetivas que abordaban los pasos relevantes del tratamiento periodontal básico. El grado de dificultad se evaluó mediante la escala Likert en 0 = ninguno, 1 = leve, 2 = moderado, 3 = alto y 4 = muy alto. Para el análisis de los datos, el grado de dificultad se dicotomizó en ninguno a leve, moderado, alto y muy alto. Resultados: participaron en el estudio 46 estudiantes matriculados en el último semestre del Curso de Odontología en el Centro Universitário da Serra Gaúcha (FSG). Marcar la pérdida de inserción (PI) fue el procedimiento descrito como alto / muy alto por los estudiantes, seguido de procedimientos de dificultad moderada, como marcar la profundidad de sondaje (PS) y raspado y alisado radicular. Sin embargo, la anestesia y el diagnóstico de gingivitis fueron los únicos procedimientos informados por todos los académicos (100\%) que no presentaban dificultades. Conclusión: De acuerdo con los resultados obtenidos en el presente estudio, se puede concluir que los procedimientos periodontales reportados como los más difíciles por los estudiantes del Curso de Odontología del Centro Universitário da Serra Gaúcha (FSG) son los procedimientos diagnósticos de periodontitis y pérdida. inserción periodontal.

Descriptores: Odontología; Enseñando; Periodoncia.

INTRODUÇÃO

A Periodontia é uma disciplina que requer riqueza de dados e conhecimentos minuciosos das estruturas de sustentação do elemento dentário. Desse modo, o dentista que se propõe a executar o tratamento periodontal, seja ele especialista ou não, deve ter domínio dos conhecimentos técnicos pertinentes à intervenção, além de possuir conhecimentos biológicos próprios da doença. Ademais, para atuar na saúde periodontal é necessário amplo conhecimento a cerca da Periodontia, o que muitas vezes exige um profissional mais capacitado e especializado nesta área ${ }^{1}$.
$\mathrm{O}$ atendimento do paciente com quadro clínico de doença periodontal pode se tornar complexo diante da falta de adequada abordagem e em função da importância dos tecidos periodontais na promoção de saúde ${ }^{2,3}$. Assim, o resultado do tratamento periodontal proposto não depende apenas do conhecimento sobre a importância dos fatores relacionados ou da remoção do biofilme dental e habilidade do profissional, mas também da preocupação do profissional frente ao desafio de educar o paciente para o controle do biofilme ${ }^{3,4}$.

Assim, no processo ensino-aprendizagem 
em Odontologia é importante a elaboração de atividades que permitam ao aluno 0 desenvolvimento progressivo da habilidade, conhecimento técnico e compreensão dos conteúdos curriculares, sendo a aprendizagem uma das mais importantes dimensões da educação ${ }^{3-5}$.

O presente estudo teve como objetivo avaliar as principais dificuldades relatadas pelos estudantes do último ano do Curso de Odontologia do Centro Universitário da Serra Gaúcha (FSG) na execução de procedimentos periodontais básicos.

MATERIAL E MÉTODO

O estudo realizado é caracterizado como observacional transversal, onde se buscou avaliar as principais dificuldades relatadas pelos estudantes do último ano do Curso de Odontologia do Centro Universitário da Serra Gaúcha (FSG) na execução de procedimentos periodontais básicos. Os dados foram coletados entre os meses de Junho de 2016 e Julho de 2016, a partir do número de alunos matriculados no último semestre do Curso de Odontologia do Centro Universitário da Serra Gaúcha (FSG). O estudo foi aprovado pelo Comitê de Ética em Pesquisa do Centro Universitário da Serra Gaúcha (CAAE 53883416.0.0000.5668).

Para se tornarem elegíveis, os participantes deveriam ter: idade superior a 18 anos e estarem matriculados no último semestre do Curso de Odontologia do Centro Universitário da Serra Gaúcha (FSG). Os participantes interessados e habilitados assinaram então, o Termo de Consentimento Livre e Esclarecido (TCLE), e responderam a um questionário estruturado composto por 14 perguntas objetivas que abordavam passos relevantes do tratamento periodontal básico. $\mathrm{O}$ questionário incluiu questões referentes às várias etapas do tratamento periodontal básico, e a percepção do grau de dificuldade foi classificada segundo a escala Likert $(0=$ nenhuma, $1=$ leve, $2=$ moderada, $3=$ alta e $4=$ muito alta). $O$ questionário utilizado neste estudo foi baseado no questionário desenvolvido em 2010 por Nagle et al. ${ }^{3}$, utilizado na pesquisa intitulada "Dificuldades relatadas por estudantes de Odontologia diante de procedimentos relacionados à Periodontia".

RESULTADOS

Participaram da pesquisa 46 alunos que estavam matriculados no último semestre do Curso de Odontologia do Centro Universitário da Serra Gaúcha (FSG). Os resultados obtidos estão representados na Tabela 1.

Entre os procedimentos periodontais básicos, marcar a perda de inserção $(\mathrm{PI})$ foi o procedimento descrito como o de dificuldade alta/muito alta pelos estudantes, seguidos por alguns procedimentos com dificuldade moderada tais como marcar a profundidade de sondagem (PS) e raspagem e alisamento radicular. Entre os procedimentos relatados com nível de dificuldade nenhuma/leve pelos acadêmicos estão o diagnóstico de gengivite e anestesiar $(100 \%)$, seguido pela radiografia inicial $(97,82 \%)$ e pela escolha do instrumento para a raspagem $(91,30 \%)$.

Tabela 1: Procedimentos clínicos periodontais e grau de dificuldade dos acadêmicos.

\begin{tabular}{l|c|c|c}
\hline \multicolumn{1}{c|}{ Procedimentos Clínicos } & $\begin{array}{c}\text { Nenhuma } \\
\text { Leve } \\
\text { N (\%) }\end{array}$ & $\begin{array}{c}\text { Moderada } \\
\text { N (\%) }\end{array}$ & $\begin{array}{c}\text { Alta } \\
\text { Muito Alta } \\
\text { N (\%) }\end{array}$ \\
\hline Diagnóstico de Gengivite & $46(100)$ & o (o) & o (o) \\
\hline Diagnóstico de Periodontite & $37(80,43)$ & $9(19,56)$ & o (o) \\
\hline Radiografia Inicial & $45(97,82)$ & o1 (2,17) & o (o) \\
\hline Visualizar PS & $29(63,04)$ & $13(28,26)$ & $4(8,69)$ \\
\hline Marcar PS & $29(63,04)$ & $11(2,17)$ & $6(13,04)$ \\
\hline Visualizar PI & $21(45,65)$ & $12(36,95)$ & $8(17,39)$ \\
\hline Marcar PI & $23(50)$ & $11(23,91)$ & $12(26,08)$ \\
\hline Anestesiar & $46(100)$ & o (o) & o (o) \\
\hline Escolha do Instrumento de RAR & $42(91,30)$ & $03(6,52)$ & $1(2,17)$ \\
\hline RAR no QSD & $28(60,86)$ & $17(36,95)$ & $1(2,17)$ \\
\hline RAR no QSE & $27(58,69)$ & $17(36,95)$ & $2(4,34)$ \\
\hline RAR no QIE & $34(73,91)$ & $11(23,91)$ & $1(2,17)$ \\
\hline RAR no QID & $34(73,91)$ & $11(23,91)$ & $1(2,17)$ \\
\hline
\end{tabular}

PS: profundidade de sondagem, PI: perda de inserção, RAR: raspagem e alisamento radicular, QSD: quadrante superior direito, QSE: quadrante superior esquerdo, QID: quadrante inferior direito, QIE: quadrante inferior esquerdo.

DISCUSSÃO

O sucesso do tratamento periodontal está relacionado diretamente a um correto diagnóstico, aliado à habilidade técnica e a preocupação do profissional frente ao desafio de educar o paciente para o correto controle do biofilme ${ }^{3}$. Quanto às dificuldades relatadas diante da necessidade de diagnóstico de gengivite e periodontite, assim como as tomadas radiográficas iniciais, observou-se que a dificuldade em diagnosticar periodontite $(19,56 \%)$ foi maior do que diagnosticar gengivite. Estes achados vão de encontro aos relatados em 2010 por Nagle et al. ${ }^{3}$, que encontraram $25 \%$ de dificuldade pelos acadêmicos em diagnosticar periodontite e $20 \%$ em diagnosticar gengivite. Em um estudo desenvolvido em 2018 por Campos e Barbosa ${ }^{4}$, os autores também relataram que os acadêmicos investigados possuem maior dificuldade na determinação do prognóstico. E que, apesar de possuírem conhecimento para tal, os mesmos se sentem inseguros quanto ao tratamento.

Os critérios de prognóstico de doença periodontal mais referidos na literatura são baseados na perda de inserção clínica (PI) associada ao sangramento à sondagem (SS) ${ }^{6-8}$. Vale ressaltar que a perda de inserção clínica é definida como a distância entre a junção 
cemento-esmalte e o fundo da bolsa e é calculada como a soma das medidas de profundidade de sondagem (PS) e recessão gengival $^{9}$. Na presente investigação, dificuldade de visualizar a PI seguida pela PS foram os procedimentos descritos como tidos certo grau de dificuldade pelos acadêmicos, o que poderia estar relacionado com a dificuldade relatada em diagnosticar periodontite. Observa-se que os procedimentos relacionados à sondagem são os de maiores dificuldades entre acadêmicos de periodontia, de um modo geral. Assim como neste, no estudo desenvolvido em 2010 por Nagle et al. $^{3}$, foi relatado que $57,5 \%$ dos estudantes apresentam algum nível de dificuldade de visualizar PI e PS.

Os benefícios associados à Raspagem e Alisamento Radicular (RAR) já são bem estabelecido na literatura ${ }^{10-13}$. A RAR são uma terapia inespecífica que tem capacidade de alterar microbiologicamente 0 ambiente subgengival e proporcionar resultados clínicos positivos, sendo este o tratamento de escolha à cura das doenças periodontais ${ }^{15}$. Em relação à essa prática, 36,95\% das maiores dificuldades ocorrem nos quadrantes superiores, sendo estas menores nos quadrantes inferiores $(23,91 \%)$, provavelmente em função do posicionamento e visibilidade obtida ${ }^{3}$.

A prática clínica tem como objetivo o desenvolvimento da habilidade técnica do aluno, através da aplicação do conhecimento teórico obtido previamente, mas essencialmente, 0 aluno deve ter consciência de suas dificuldades, de seus limites e da necessidade de contínuo aprendizado $^{3,15}$.

CONCLUSÃO

De acordo com os resultados obtidos no presente estudo, pode-se concluir que os procedimentos periodontais relatados como os de maiores dificuldades, pelos estudantes do Curso de Odontologia do Centro Universitário da Serra Gaúcha (FSG), são os procedimentos relacionados com o diagnóstico de periodontite que se dão através da visualização da perda de inserção e profundidade de sondagem.

\section{REFERÊNCIAS}

1. Lotufo R. Roberto Lotufo: entrevista. Dental Press Perio Implant. 2007;1(4):15-24.

2. Saini R, Marawar PP, Shete S, Saini S. Periodontitis, a true infection. J Glob Infect Dis. 2009;1(2):149-50.

3. Nagle MM, Piveta ACG, Ricci WA, Montadon AFB. Dificuldades relatadas por estudantes de odontologia diante de procedimentos relacionados à periodontia. Rev ABENO. 2010; 10(1):37-41.
4. Campos JR, Barbosa FI. Diagnóstico Periodontal: conhecimentos e atitudes de estudantes de Odontologia. Arq Odontol. 2018, $54: 1-7$

5. Gotteher NR, Martin JL. A future for periodontal therapy. Dent Today. 2010; 29(2):154,156-57.

6. Dias Sobrinho J. Avaliação - política e reformas da educação superior. São Paulo: Cortez; 2003.

7. López NJ, Smith PC, Gutierrez J. Periodontal therapy may reduce the risk of preterm low birth weight in women with periodontal disease: a randomized controlled trial. J Periodontol. 2002; 73(8):911-24.

8. Cruz SS, Costa MCN, Gomes-Filho IS, Vianna MIP. Doença periodontal materna como fator associado ao Baixo Peso ao Nascer. Rev Saúde Pública. 2005;39(2).

9. Gomes F. Critérios para o Diagnóstico Clínico da Doença Periodontal. J Bras Clín Odontol Integr Saúde Bucal Colete. 2005;9(49):88-9.

10. Susin C, Dalla Vecchia CF, Oppermann RV, Haugejorden O, Albandar JM. Periodontal attachment loss in an urban population of Brazilian adults: effect of demographic, behavioral, and environmental risk indicators. J Periodontol. 2004;75(7):1033-41.

11. Haffajee AD, Cugini MA, Dibart SS, Kent RL, Socransky SS. The effect of SRP on the clinical and microbiological parameters of periodontal diseases. J Clin Periodontol. 1997;24:324-34.

12. Cugini MA, Haffajee AD, Smith $C$, Kent $R L$, Socransky SS. The effect of scaling and root planning on the clinical and microbiological parameters of periodontal diseases: 12 mounths results. J Clin Periodontol. 2000;27(1): 30-6.

13. Cobb CM. Clinical significance of non-surgical periodontal therapy: an evidence based perspective of scaling and root planning. J Clin Periodontol. 2002;29(Suppl 2):6-16.

14. Carvalho LH, D'Avila GB, Leão A, Haffajee AD, Socransky SS, Feres M. Scaling and root planing, systemic metronidazole and professional plaque removal in the treatment of chronic periodontitis in a Brazilian population. II. Microbiological results. J Clin Periodontol. 2005;32(4):406-11.

15.Bosi SV, Feron L, Marcon J, Butze JP. Perfil dos pacientes atendidos na clínica de periodontia do Centro Universitário da Serra Gaúcha (FSG). Arch Health Invest. 2018;7(6): 233-36. 


\section{CONFLITO DE INTERESSES}

Os autores declaram não haver conflitos de interesse

\section{AUTOR PARA CORRESPONDÊNCIA}

\section{Juliane Pereira Butze}

Curso de Odontologia, Centro Universitário da Serra

Gaúcha - FSG

Rua Os Dezoito do Forte, 2366. Bairro São Pelegrino.

95020-472 Caxias do Sul - RS, Brasil

Telefone: (54) 21016026/ (51) 981344083.

E-mail: juliane.butze@fsg.edu.br 